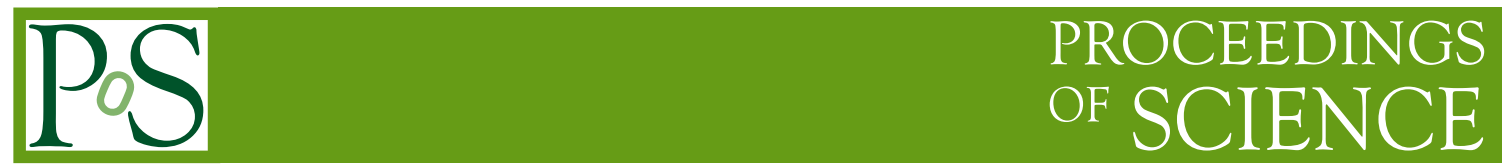

\title{
A mass preconditioning for lattice Dirac operators
}

\author{
Nazario Tantalo*† \\ Rome University "Tor Vergata" and INFN sez. Rome "Tor Vergata" \\ E-mail: nazario.tantalo@roma2.infn.it
}

In a recent publication [1] we have proposed a preconditioning of the Dirac operator based on the factorisation of a predefined function related to the decay of the propagator with the distance. In this talk we discuss this numerical technique by arguing that it can be profitably used in order to improve the accuracy of correlators involving heavy quarks at large distances and eventually to accelerate the computation of light quark propagators.

The XXVIII International Symposium on Lattice Field Theory, Lattice2010

June 14-19, 2010

Villasimius, Italy

\footnotetext{
*Speaker.

† based on work done in collaboration with G.M. de Divitiis and R. Petronzio
} 


\section{Introduction}

In order to calculate observables in a typical lattice QCD simulations one needs to solve numerically a linear system of the form

$$
\sum_{y}(D[U]+M)_{x, y} S(y)=\eta(x)
$$

where $D[U]$ is the chosen discretization of the massless interacting Dirac operator, $M$ is the quark mass in lattice units, $\eta(x)$ is a source vector that is different from zero on a single time-slice (that without any loss we shall assume to be at $\left.x_{0}=0\right)$. The solution $S(y)$ is obtained by iterative numerical algorithms, solvers, devised to invert so-called sparse matrices, i.e. those matrices that result from the discretization of differential equations by finite differences methods. Roughly speaking (see ref. [2] for a detailed review and for an updated list of references) a solver gets the solution by applying the sparse matrix a few times, by performing a bit of linear algebra and by checking that the condition

$$
\left|\sum_{y}(D[U]+M)_{x, y} S^{n}(y)-\eta(x)\right|<r
$$

is satisfied. Here $S^{n}(y)$ is the tentative solution at iteration number $n$, the norm is any good norm in field space and $r$, the residue, is the global numerical accuracy requested for the solution. Typically $r$ is a small number of the order of the arithmetic precision allowed by the computer architecture.

For light quarks the matrix $(D[U]+M)_{x, y}$ is badly conditioned and its numerical inversion requires a big number of iterations. At the other extreme, the number of iterations required for heavy quark masses is small but there may be problems with the numerical accuracy resulting for the time-slices far away from the source $\left(y_{0} \gg 0\right)$. Indeed eq. (1.2) is a global condition while for heavy quark propagators the time-slices far away from the source are exponentially suppressed by a factor of the order of $\exp \left(-M y_{0}\right)$ and give a negligible contribution to the norm on the left side of eq. (1.2). When this problem arises one cannot trust numerical results at large times and it becomes impossible to extract physical informations by fitting the leading exponentials contributing to correlation functions. In the rest of this talk we shall illustrate a preconditioning technique that can be profitably used in alleviating both these difficulties.

\section{Heavy quark propagators decay too fast!}

In the following we shall work with the $O(a)$-improved Wilson lattice Dirac Operator but the numerical problems that we address arise also with alternative discretisations of the continuum action and the proposed solution can as well be easily implemented in those cases. We first illustrate the numerical problem. It arises when the product of the mass of the quark with the size of the lattice in the time direction is big. As an example, we have calculated the correlation function

$$
C_{P P}\left(y_{0}\right)=-\sum_{\vec{y}} \operatorname{tr}\left[S^{\dagger}(y) S(y)\right]=-\sum_{\vec{y}} \operatorname{tr}\left|S\left(\vec{y}, y_{0}\right)\right|^{2}
$$

by solving eq. (1.1) for a heavy quark propagator of mass $M \simeq 0.5$ in the free theory for different choices of the residue. More precisely the red points in Figure 1 have been obtained with a residue 


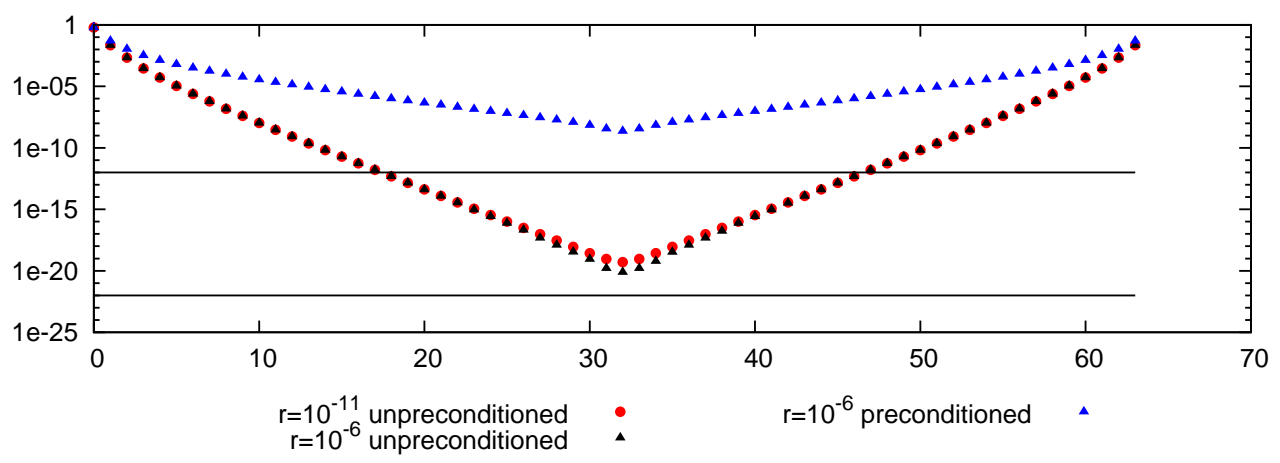

Figure 1: The red points correspond to the correlation function $-C_{P P}\left(y_{0}\right)$ obtained by inverting the lattice Dirac equation in the free theory with $M \simeq 0.5$ and with a residue $r=10^{-11}$. The black points correspond to the same quantity but have been obtained with a residue $r=10^{-6}$. The blue points correspond to the correlation function $-C_{P P}^{\prime}\left(y_{0}\right)$ obtained by solving the preconditioned lattice Dirac equation with $M \simeq 0.5$ and $\alpha_{0}=0.4$. The two black lines correspond to $r^{2}$ for the two values of the residue used in the calculations. We use logarithmic scale on the $y$-axis.

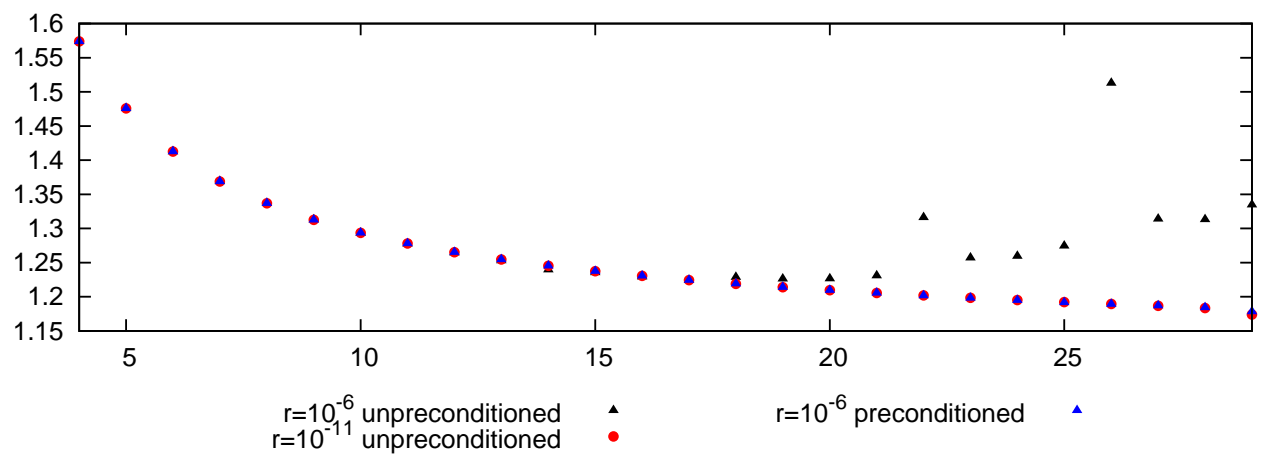

Figure 2: The red and black sets of points are the effective masses of the corresponding correlators shown in the previous figure. The blue set of points is the effective mass of the corresponding correlator in the top panel multiplied for the restoration factor as explained in the text.

$r=10^{-11}$ while the black points with a residue $r=10^{-6}$ and the two black lines correspond to the squares of these two values of $r$. As is clearly visible from Figure 1, and from Figure 2 where we show the effective masses of the correlations shown in Figure 1, the black points start to deviate from the red ones for $y_{0} \simeq 18$, i.e. when the correlator, which in this case is just the square module of the propagator (see eq. (2.1)), becomes smaller than the square of the "loose" residue $r=10^{-6}$. That's what we mean by saying that "heavy quark propagators decay too fast!".

If the time extent of the lattice is not too large the problem can be solved by brute force by lowering the residue and the results obtained in the preset case with $r=10^{-11}$ can be considered as exact. If instead the time extent of the lattice is rather large the brute force approach cannot be considered because the required residues would be smaller than what is allowed on double-precision architectures, even in the case of moderately heavy quarks. In the case under consideration, by 
choosing a loose precision, i.e. a residue $r=10^{-6}$, we make the numerical problem evident and we show that also such an "extreme" situation can be recovered by using our proposal. Moreover, we notice that a residue $r=10^{-6}$ is the smallest allowed on single-precision architectures that presently are considerably much faster than double-precision ones.

We now come to the proposed solution. We redefine the quark fields and the propagators as follows

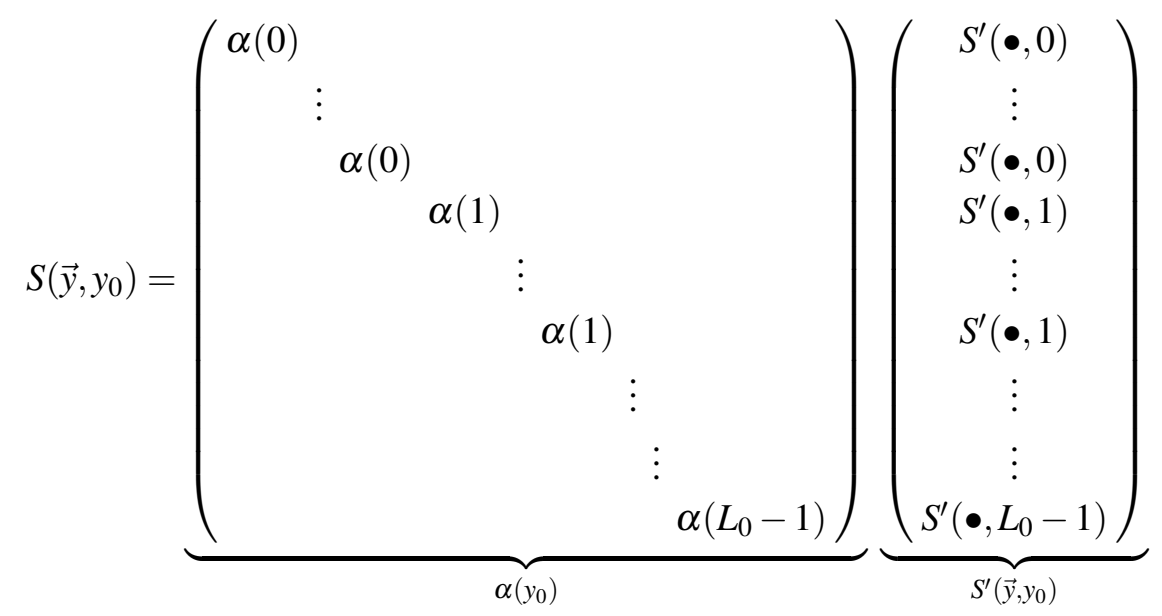

$$
\begin{aligned}
& \eta(y)=\alpha\left(y_{0}\right) \eta^{\prime}(y)
\end{aligned}
$$

The function/matrix $\alpha\left(y_{0}\right)$ is diagonal in colour, Dirac and space indexes and must be invertible. Once the previous expressions are inserted in eq. (1.1) one gets the preconditioned system

$$
\sum_{y}\left(D^{\prime}[U]+M\right)_{x, y} S^{\prime}(y)=\eta^{\prime}(x)
$$

that is solved numerically in place of eq. (1.1). In order to write the preconditioned Dirac operator it is sufficient to modify the forward and backward lattice covariant derivatives in the time direction according to

$$
\begin{gathered}
\nabla_{0} S(y)=U_{0}(y) S(y+\hat{0})-S(y) \quad \longrightarrow \frac{\alpha\left(y_{0}+1\right)}{\alpha\left(y_{0}\right)} U_{0}(y) S^{\prime}(y+\hat{0})-S^{\prime}(y) \\
\nabla_{0}^{\dagger} S(y)=S(y)-U_{0}^{\dagger}(y-\hat{0}) S(y-\hat{0}) \quad \longrightarrow \quad S^{\prime}(y)-\frac{\alpha\left(y_{0}-1\right)}{\alpha\left(y_{0}\right)} U_{0}^{\dagger}(y-\hat{0}) S^{\prime}(y-\hat{0})
\end{gathered}
$$

If as in the case of Figure $1 S(y)$ satisfies anti-periodic boundary conditions along the time direction, it follows from eq. (2.2) that

$$
S\left(y+L_{0} \hat{0}\right)=-S(y) \quad \longrightarrow \quad S^{\prime}\left(y+L_{0} \hat{0}\right)=-\frac{\alpha\left(y_{0}\right)}{\alpha\left(y_{0}+L_{0} \hat{0}\right)} S^{\prime}(y)
$$

The blue points in Figure 1 correspond to the correlation function

$$
C_{P P}^{\prime}\left(y_{0}\right)=-\sum_{\vec{y}} \operatorname{tr}\left[\left(S^{\prime}\right)^{\dagger}(y) S^{\prime}(y)\right]
$$




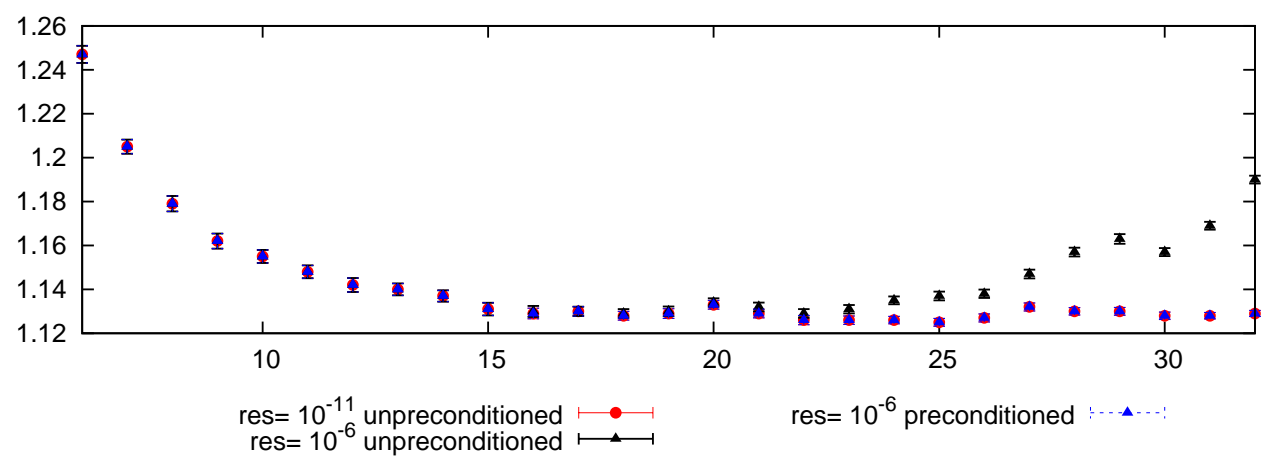

Figure 3: The red points correspond to the effective mass of the correlation function $-C_{P P}\left(y_{0}\right)$ obtained by inverting the lattice Dirac equation in the interacting theory with $\mathrm{am}_{h}^{p c a c} \simeq 0.35$ and with a residue $r=10^{-11}$. The black points correspond to the same quantity but have been obtained with a residue $r=10^{-6}$. The blue points correspond to the effective mass of the restored correlation function $-C_{P P}^{\prime}\left(y_{0}\right)$ obtained by solving the preconditioned lattice Dirac equation with $a m_{h}^{p c a c} \simeq 0.35$ and $\alpha_{0}=0.4$.

obtained by solving eq. (2.3) with the loose residue $r=10^{-6}$ but after having factorized the function

$$
\alpha\left(y_{0}\right)=\cosh \left[\alpha_{0}\left(y_{0}-L_{0} / 2\right)\right]
$$

with $\alpha_{0}=0.4$. As expected, the preconditioned correlator stays above the line of the loose precision residue and the "exact" result can be back recovered as follows

$$
C_{P P}\left(y_{0}\right)=\left[\alpha\left(y_{0}\right)\right]^{2} C_{P P}^{\prime}\left(y_{0}\right)
$$

In Figure 2 the blue points correspond to the effective mass of the preconditioned correlator after the "restoration" of eq. (2.8) and fall exactly on top of the red ones in spite of the fact that they have been obtained with the same loose precision that affected the non preconditioned black points.

In Figure 3 we show the same plot as in Figure 2 but in the interacting theory. The particular gauge ensemble used correspond to the entry E5 in Table 1. The size of the lattice is $L_{0} L_{1} L_{2} L_{3}=64 \times 32^{3}$ and the hopping parameter of the sea quarks is $k_{\text {sea }}=0.13625$ corresponding to a PCAC quark mass of about $a m_{\text {sea }}^{P C A C} \simeq 0.07$. The data shown in Figure 3 correspond to a pseudoscalar-pseudoscalar correlator, as in the free theory case, of two degenerate heavy quarks with hopping parameters $k_{h}=0.125$ corresponding to a PCAC quark mass of about $a m_{h}^{P C A C} \simeq 0.35$. The unpreconditioned correlators decay approximately as fast as as in the free theory case and from the difference of the black (unpreconditioned, $r=10^{-6}$ ) and red (unpreconditioned, $r=10^{-11}$ ) sets of data we see the same distortion of Figure 2. The blue points have been obtained by solving eq. (2.3) after having factorized $\alpha\left(y_{0}\right)$ with $\alpha_{0}=0.4$ and by restoring the results according to eq. (2.8). Also in the interacting theory the blue points are identical to red points though they have been obtained with the same loose residue $r=10^{-6}$ used to obtain the black points.

We close this section by observing that our preconditioning technique may be particularly useful when working with the Schrödinger Functional (SF) [3, 4] formulation of the theory. In this case, contrary to the case of periodic boundary conditions along the time direction, the correlators decay exponentially over the whole time extent of the lattice and one has to choose very small 


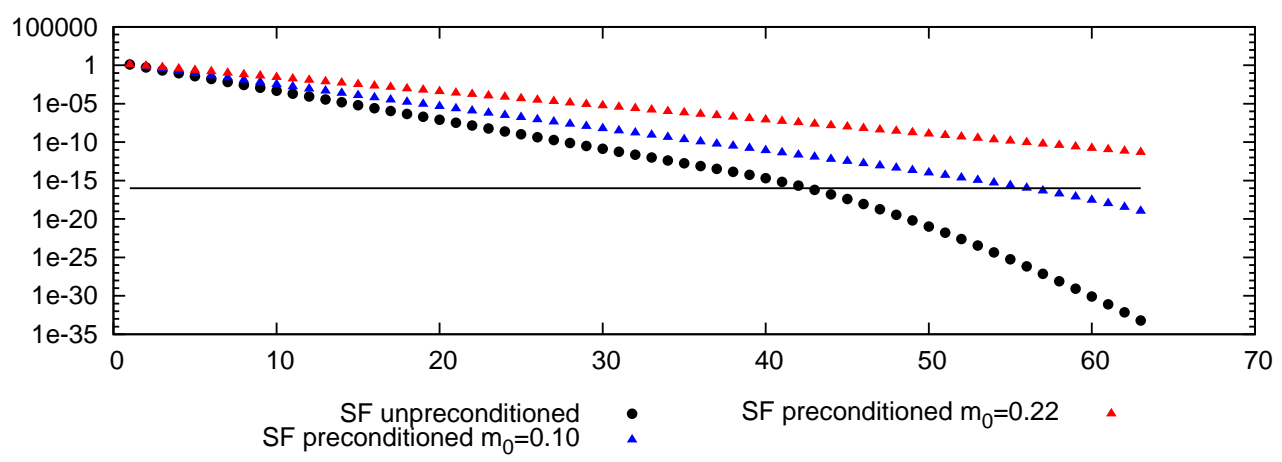

Figure 4: The black points correspond to the correlation function $-C_{P P}\left(y_{0}\right)$ obtained by inverting the lattice Dirac equation in the Schrödinger Functional free theory with $M \simeq 0.5$ and with a residue $r=10^{-8}$. The blue and red points correspond to the preconditioned quantity (before restoration) obtained with $\alpha\left(x_{0}\right)=$ $\exp \left(-\alpha_{0} x_{0}\right)$ at different values of $\alpha_{0}$. The red points stay above the residue threshold (black solid line) at all times and fall exactly on a straight-line, the theoretical prediction for this quantity by using logarithmic scale on the $y$-axis.

residues also in computing relatively light quark propagators. In Figure 4 we show the correlator $-C_{P P}\left(x_{0}\right)$ in free theory on a lattice with SF boundary conditions in the time direction $\left(L_{0}=64\right)$ : this quantity must be a straight line in logarithmic scale while starts to bend in the unpreconditioned case (black points) at large times when the propagator becomes smaller than the residue (here $\left.r=10^{-8}\right)$. By using our preconditioning with $\alpha\left(x_{0}\right)=\exp \left(-\alpha_{0} x_{0}\right)$ and an appropriate value of $\alpha_{0}$ it is possible to perform a safe inversion also in the SF case. We have observed the same phenomenology also in the SF interacting theory.

\section{Preconditioning light quark propagators}

The ideas developed and discussed in the previous section can be used to accelerate the numerical calculation of light quark propagators by generalising eq. (2.2) as follows

$$
\begin{aligned}
S(y) & =\beta\left(y_{0}, y_{1}, y_{2}, y_{3}\right) S^{\prime}(y) \\
\eta(y) & =\beta\left(y_{0}, y_{1}, y_{2}, y_{3}\right) \eta^{\prime}(y) \\
\beta\left(y_{0}, y_{1}, y_{2}, y_{3}\right) & =\prod_{\mu=0}^{3} \alpha\left(y_{\mu}\right)=\prod_{\mu=0}^{3} \frac{1}{\cosh \left[\alpha_{0}\left(y_{\mu}-L_{\mu} / 2\right)\right]}
\end{aligned}
$$

The preconditioned lattice Dirac operator can be obtained as easily as before by changing accordingly the covariant derivatives in all directions as done in eqs. (2.4) and (2.5) for the time direction only. An important difference of the present case with respect to the one discussed in the previous section is that the restoration of the true propagator must be performed before making the contractions needed to build correlation functions by using the first of eqs. (3.1). 


\begin{tabular}{ccccccc} 
& $\beta$ & $L_{0} L_{1} L_{2} L_{3}$ & $k_{\text {sea }}$ & $r$ & $\alpha_{0}$ & iterations \\
\hline & & & & & & \\
$D 5$ & 5.3 & $48 \times 24^{3}$ & 0.13625 & $10^{-11}$ & 0.0 & 175 \\
$D 5$ & 5.3 & $48 \times 24^{3}$ & 0.13625 & $10^{-11}$ & 0.4 & 141 \\
& & & & & & \\
$E 3$ & 5.3 & $64 \times 32^{3}$ & 0.13605 & $10^{-10}$ & 0.0 & 99 \\
$E 3$ & 5.3 & $64 \times 32^{3}$ & 0.13605 & $10^{-10}$ & 0.2 & 78 \\
$E 3$ & 5.3 & $64 \times 32^{3}$ & 0.13605 & $10^{-10}$ & 0.4 & 69 \\
$E 4$ & 5.3 & $64 \times 32^{3}$ & 0.13610 & $10^{-10}$ & 0.0 & 115 \\
$E 4$ & 5.3 & $64 \times 32^{3}$ & 0.13610 & $10^{-10}$ & 0.2 & 91 \\
$E 4$ & 5.3 & $64 \times 32^{3}$ & 0.13610 & $10^{-10}$ & 0.4 & 81 \\
$E 5$ & 5.3 & $64 \times 32^{3}$ & 0.13625 & $10^{-10}$ & 0.0 & 194 \\
$E 5$ & 5.3 & $64 \times 32^{3}$ & 0.13625 & $10^{-10}$ & 0.2 & 153 \\
$E 5$ & 5.3 & $64 \times 32^{3}$ & 0.13625 & $10^{-10}$ & 0.4 & 141 \\
& & & & & & \\
\hline
\end{tabular}

Table 1: Gauge configurations have been generated with $n_{f}=2$ dynamical $O(a)$-improved Wilson quarks with $c_{s w}=1.90952$. The figures in the last column correspond to the average of the number of iterations required to invert the Dirac equation in the unitary theory by using the SAP+GCR inverter for several values of the preconditioning parameter $\alpha_{0}$. The values corresponding to $\alpha_{0}=0.0$ have been obtained without using our preconditioning technique.

By judiciously choosing the parameter $\alpha_{0}$ it is possible to change the condition number of the preconditioned system without compromising the numerical accuracy of the solution and in Table 1 we quantify the gain in computational time that can be achieved by showing the number of iterations of the SAP+GCR solver required to solve the lattice Dirac equation for light quarks with and without our preconditioning. The SAP+GCR solver has been introduced and explained in details by the author in ref. [5]. The parameters of the gauge ensembles used to perform this test are given in the table and have been generated within the CLS agreement [6]. We thanks our colleagues of the CLS community for sharing the efforts required to generate the dynamical gauge ensembles used in this study.

\section{References}

[1] G. M. de Divitiis, R. Petronzio, N. Tantalo, Phys. Lett. B692 (2010) 157-160. [arXiv:1006.4028 [hep-lat]].

[2] M. Luscher, arXiv:1002.4232 [hep-lat].

[3] M. Luscher, R. Narayanan, P. Weisz and U. Wolff, Nucl. Phys. B 384 (1992) 168 [arXiv:hep-lat/9207009].

[4] S. Sint, Nucl. Phys. B 421 (1994) 135 [arXiv:hep-lat/9312079].

[5] M. Luscher, Comput. Phys. Commun. 156 (2004) 209 [arXiv:hep-lat/0310048].

[6] https://twiki.cern.ch/twiki/bin/view/CLS/WebHome 